\title{
Neuroimaging in Parkinson's Disease
}

\author{
David J. Brooks \\ Medical Research Council Clinical Sciences Center and Division of Neuroscience, Faculty of Medicine, Imperial College, \\ Hammersmith Hospital, London W12 0NN, United Kingdom
}

\begin{abstract}
Summary: In this review, the potential role of positron emission tomography and single photon emission computed tomography as biological markers for diagnosing and following the progression of Parkinson's disease (PD) is discussed. Their value for assessing the efficacy of putative neuroprotective agents in PD and for revealing the pharmacological changes underlying the symptomatology and complications of this dis-
\end{abstract}

order is also considered. It is concluded that in the future functional imaging will provide a valuable adjunct to clinical assessment when judging the efficacy of putative neuroprotective approaches to PD. Key Words: Positron emission tomography, SPECT, Parkinson's disease, dopamine, neuroprotection, progression.

\section{INTRODUCTION}

The development of high-field magnetic resonance imaging (MRI) with three-dimensional (3D) volumetric acquisitions, gray and white matter suppressing inversion recovery sequences and diffusion-weighted imaging (DWI) has led to a more important role for structural imaging in the diagnosis of parkinsonian disorders. High-field MRI can now detect substantia nigra compacta pathology in the majority of idiopathic Parkinson's disease (PD) patients and, in principle, measure the volume of this nucleus. ${ }^{1,2}$ In atypical parkinsonian syndromes abnormal putamen and pontine signal may be present on $\mathrm{T} 2$ or diffusion-weighted images along with atrophy of these structures, cortex and midbrain. $.^{3-5} \mathrm{Al}-$ ternatively, radiotracer-based functional imaging provides a sensitive means of detecting and characterizing the regional changes in brain metabolism and receptor binding associated with parkinsonian disorders. It can be of diagnostic value and also provide a sensitive means of detecting subclinical disease in subjects at risk for degenerative disorders and of objectively following disease progression. Positron emission tomography (PET) has the highest sensitivity, being able to detect femtomolar levels of positron-emitting radioisotopes at a spatial resolution of 3-5 $\mathrm{mm}$ and correct for scatter. It allows

Address correspondence and reprint requests to David J. Brooks, Imperial College London, Cyclotron Building, Hammersmith Hospital, Du Cane Rd, London W12 0NN, UK. E-mail: david.brooks@csc.mrc.ac.uk. quantitative in vivo examination of alterations in regional cerebral blood flow, glucose, oxygen, dopa metabolism, and brain receptor binding. Single photon emission tomography (SPECT) is less sensitive and is unable to correct for scatter but is more widely available and provides measures of $\mathrm{rCBF}$ and receptor binding.

\section{THE PATHOLOGY OF PD}

Neuronal loss in PD targets the dopamine cells in the substantia nigra in association with the formation of intracellular Lewy inclusion bodies. ${ }^{6}$ Serotonergic cells in the median raphe, noradrenergic cells in the locus ceruleus, and cholinergic cells in the nucleus basalis are also involved to a lesser extent as are other pigmented and brainstem nuclei. Loss of cells from the substantia nigra in PD results in profound dopamine depletion in the striatum, with lateral nigral projections to putamen being most affected. ${ }^{7,8}$ This clinically manifests as a combination of bradykinesia, rigidity, and tremor that is characteristically asymmetric in onset. Lewy bodies are also found in neurons of the anterior cingulate and frontal, parietal, and temporal association cortex of most nondemented PD cases at postmortem. ${ }^{9}$ Dementia is twice as prevalent in PD compared with age-matched controls. ${ }^{10}$ Currently it remains uncertain whether dementia associated with cortical Lewy bodies and PD represent opposite ends of a spectrum. Dementia of Lewy body type has overlapping features with Alzheimer's disease, although it is said to be associated with a higher prevalence of 


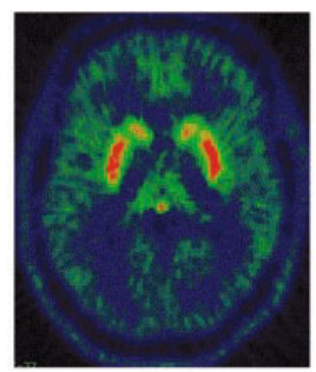

Normal

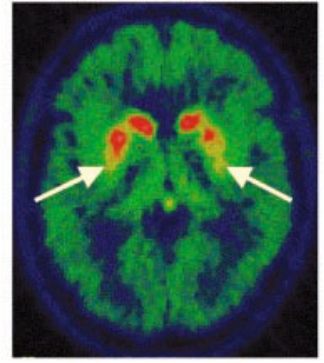

Early hemi-PD

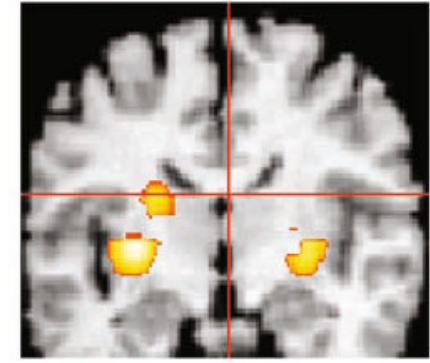

SPM

FIG. 1. ${ }^{18} \mathrm{~F}$-dopa PET. ${ }^{18} \mathrm{~F}$-dopa uptake in a normal subject and early hemi-PD patient. The PD patient shows bilateral posterior putamen loss of signal. Statistical parametric mapping localizes significant reductions in ${ }^{18} \mathrm{~F}$-dopa uptake in the caudate and putamen contralateral and the posterior putamen ipsilateral to the affected limb in a group of hemi-PD cases (picture courtesy of J. Rakshi, MRC Clinical Sciences Center and Division of Neuroscience, Faculty of Medicine, Imperial College London, UK).

fluctuating confusion, hallucinations, early onset rigidity, and gait difficulties. ${ }^{11}$

\section{IMAGING THE PRESYNAPTIC DOPAMINERGIC SYSTEM}

The function of dopamine terminals in PD can be examined in vivo in several ways: first, terminal dopa decarboxylase (DDC) activity can be measured with ${ }^{18} \mathrm{~F}$-dopa PET. Second, the availability of presynaptic dopamine transporters (DAT) can be assessed with tropane-based PET and SPECT tracers. Third, vesicle monoamine transporter density in dopamine terminals can be examined with ${ }^{11} \mathrm{C}$-dihydrotetrabenazine (DHTBZ) PET.

In early hemiparkinsonian cases ${ }^{18} \mathrm{~F}$-dopa PET shows bilaterally reduced putamen tracer uptake (FIG. 1), with activity being most depressed by around 50\% in the caudal putamen contralateral to the affected limbs and by $25 \%$ in the "asymptomatic" putamen contralateral to clinically unaffected limbs. ${ }^{12,13}$ PD patients with established disease show a $60-80 \%$ loss of specific putamen ${ }^{18} \mathrm{~F}$-dopa uptake in life, ${ }^{14,15}$ in line with the reported loss of ventrolateral nigra compacta cells but less than the 95\% loss of putamen dopamine postmortem. ${ }^{16}$ These findings suggest that striatal dopamine terminal DDC activity may be relatively upregulated in $\mathrm{PD}$, presumably to boost dopamine turnover by remaining neurons.

It is known that the pathology of PD is not uniform and ventrolateral nigral dopaminergic projections to the dorsal putamen are more affected than dorsomedial projections to the head of caudate. ${ }^{7}{ }^{18} \mathrm{~F}$-dopa PET reveals that in patients with unilateral PD (Hoehn and Yahr stage 1) contralateral dorsal posterior putamen dopamine storage is first reduced. ${ }^{12}$ As all limbs become clinically affected, ventral and anterior putamen and dorsal caudate dopaminergic function also become involved. Finally, when PD is well advanced, the ventral head of caudate ${ }^{18} \mathrm{~F}$-dopa uptake starts to fall.

Not all dopamine fibers degenerate in early PD. Nigrostriatal projections comprise the densest dopamine pathway but there is also a nigro-internal pallidal pathway with $20 \%$ of their density. ${ }^{17}$ The striatum is the main input and the globus pallidus interna (GPi) is the main output nucleus of the basal ganglia, and the dopamine system modulates the function of both these structures. While putamen ${ }^{18} \mathrm{~F}$-dopa uptake is reduced by at least $30 \%$ at the onset of parkinsonian rigidity and bradykinesia, GPi ${ }^{18} \mathrm{~F}$-dopa uptake is initially increased by $40 \%{ }^{18}$ This increased uptake subsequently falls below normal levels as disease advances and loss of GPi ${ }^{18} \mathrm{~F}$-dopa upregulation coincides with the presence of fluctuating responses to levodopa, suggesting that both putamen and pallidum tonic dopamine release is required to facilitate fluent and efficient limb movements.

There are now a number of tropane-based radioligands available for measuring DAT binding on nigro-striatal terminals and so providing a measure of their integrity. PET tracers include ${ }^{11} \mathrm{C}-\mathrm{CFT},{ }^{18} \mathrm{~F}-\mathrm{CFT}$, and ${ }^{11} \mathrm{C}-\mathrm{RTI}-32$, which bind to both dopamine and noradrenaline reuptake sites. ${ }^{19-21}$ SPECT tracers available include ${ }^{123} \mathrm{I}-\beta$-CIT, ${ }^{123} \mathrm{I}$-FP-CIT, ${ }^{123} \mathrm{I}$-altropane, and ${ }^{99 \mathrm{~m}} \mathrm{Tc}$-TRODAT- $1 .{ }^{22-26}$ ${ }^{123} \mathrm{I}-\beta$-CIT gives the highest striatal:cerebellar uptake ratio of these SPECT tracers but this reflects a lower cerebellar nonspecific uptake rather than a higher striatal specific uptake compared with the alternatives. ${ }^{123} \mathrm{I}-\beta$ CIT binds nonselectively to dopamine, noradrenaline, and serotonin transporters and has the disadvantage that it takes $24 \mathrm{~h}$ to equilibrate throughout the brain following intravenous injection, therefore scanning has to be delayed until the following day. For this reason, SPECT tracers such as ${ }^{123} \mathrm{I}$-FP-CIT, and ${ }^{123} \mathrm{I}$-altropane have come into vogue as, despite their lower and time-dependent striatal:cerebellar uptake ratios, a diagnostic scan can be performed within 2-3 $\mathrm{h}$ of tracer injection. Most recently a technetium-based tropane tracer, ${ }^{99 \mathrm{~m}} \mathrm{Tc}-\mathrm{TRO}-$ DAT-1, has been developed. This gives a lower 2:1 striatal:cerebellar uptake ratio than the ${ }^{123} \mathrm{I}$-based tracers and is less well extracted by the brain but has the advantage that it is potentially available in kit form. 
All of the above PET and SPECT tracers differentiate clinically probable early PD from normal subjects or essential tremor patients with a sensitivity of around 90\%. Given this, a positive PET or SPECT scan is valuable for supporting a diagnosis of PD where there is diagnostic doubt. It is less clear, however, whether a negative PET or SPECT scan can completely exclude this diagnosis (see later).

Putamen uptake of PET and SPECT dopaminergic tracers shows an inverse correlation with limb bradykinesia and rigidity in PD but not with rest tremor severity. ${ }^{23,27-29}$ Relative to putamen binding of the dopamine vesicle transporter marker, ${ }^{11} \mathrm{C}$-DHTBZ, it has been shown that specific ${ }^{18} \mathrm{~F}$-dopa uptake is raised and that binding of the DAT marker ${ }^{11} \mathrm{C}$-methylphenidate is reduced in PD. ${ }^{30}$ This finding suggests the presence of compensatory mechanisms in PD, DDC activity being upregulated, and dopamine transporter binding downregulated. Such a situation would make physiological sense because increased dopamine turnover and decreased reuptake would help to preserve synaptic transmitter levels in a dopamine deficiency syndrome.

In PD there is loss not only of dopamine but also serotonin projections. Median raphe serotonin $\mathrm{HT}_{1 \mathrm{~A}}$ binding in the midbrain, measured with ${ }^{11} \mathrm{C}-\mathrm{WAY} 100635$ PET, reflects the functional integrity of serotonergic cell bodies. In PD there is a mean $25 \%$ loss of median raphe HT1A binding and individual levels correlate with severity of rest tremor. ${ }^{31}$ This finding suggests that midbrain tegmentum pathology involving serotonin projections may be more relevant to the etiology of PD tremor than loss of nigrostriatal projections.

\section{PRECLINICAL PD}

It has been estimated from postmortem studies that $10 \%$ of elderly subjects may have incidental Lewy body pathology. ${ }^{32}$ This exceeds the prevalence of PD 10- to 15 -fold and subjects most likely to be at risk include relatives of patients with the disorder. In one series investigating $\mathrm{PD}$ kindreds with ${ }^{18} \mathrm{~F}$-dopa PET, $25 \%$ of asymptomatic adult relatives were found to have subclinical putamen loss of dopamine terminal function. ${ }^{33}$ Three of eight of these asymptomatic relatives with reduced putamen ${ }^{18} \mathrm{~F}$-dopa uptake who were followed longitudinally over 5 years subsequently developed clinical parkinsonism.

${ }^{18}$ F-dopa PET findings for adult asymptomatic cotwins of idiopathic sporadic PD patients have also been reported. In one series $55 \%$ of monozygotic (MZ) cotwins compared with $18 \%$ of dizygotic (DZ) co-twins had levels of putamen ${ }^{18} \mathrm{~F}$-dopa uptake that were reduced more than 2.5 SD below the normal mean $(p<0.03){ }^{34}$ The finding of a significantly higher concordance for dopaminergic dysfunction in MZ compared with DZ PD co-twins supports a genetic contribution toward this apparently sporadic disorder. When a subgroup of these co-twins were scanned serially over 4 years all 10 of the asymptomatic MZ co-twins scanned showed a decrease in putamen ${ }^{18} \mathrm{~F}$-dopa uptake (mean $4.5 \%$ annual loss) and two became symptomatic while the nine asymptomatic DZ co-twins showed no significant change in their putamen ${ }^{18} \mathrm{~F}$-dopa uptake.

The association of young-onset L-dopa-responsive parkinsonism with homozygosity or compound heterozygosity for parkin gene mutations is now well accepted. In contrast to idiopathic PD, however, parkin disease is not associated with Lewy body formation in the majority of cases. For their level of clinical disability these cases show severe loss of striatal ${ }^{18} \mathrm{~F}$-dopa uptake with greater caudate involvement than is seen in idiopathic PD. ${ }^{35-37} \mathrm{It}$ is now recognized that heterozygote parkin mutation carriers can be at risk for late-onset PD, particularly if exon 7 is involved. In one series asymptomatic heterozygote parkin mutation carriers have been studied with ${ }^{18}$ F-dopa PET. ${ }^{35}$ Individual carriers had low normal levels of putamen ${ }^{18} \mathrm{~F}$-dopa storage, whereas as a group, mean putamen ${ }^{18} \mathrm{~F}$-dopa uptake was significantly reduced by $30 \%$ although it did not reach symptomatic levels.

\section{MICROGLIAL ACTIVATION IN PARKINSON'S DISEASE}

Microglia constitute 10-20\% of white cells in the brain and form its natural defense mechanism. They are normally in a resting state but local injury causes them to activate and swell, expressing histocompatibility locus antigens on the cell surface and releasing cytokines. The mitochondria of activated but not resting microglia express peripheral benzodiazepine (BDZ) sites that may have a role in preventing apoptosis via membrane stabilization. PK11195 is an isoquinoline that binds selectively to peripheral BDZ sites and thus ${ }^{11} \mathrm{C}-\mathrm{PK} 11195$ provides an in vivo PET marker of glial activation. ${ }^{38}$

Loss of substantia nigra neurons in PD is known to be associated with microglial activation. ${ }^{39}{ }^{11} \mathrm{C}-\mathrm{PK} 11195$ PET has been used to study microglial activation in PD and has detected increased signal in both the nigra and pallidum (FIG. 2). ${ }^{40}$ The nigral ${ }^{11} \mathrm{C}$-PK11195 uptake is likely to reflect local degeneration, whereas the pallidal signal may result from the excess glutamate release from subthalamic projections that results as a consequence of dopamine deficiency. It is likely that in the future, putative neuroprotective and anti-inflammatory agents will be tested to determine whether they can reverse in vivo the microglial activation associated with PD. 

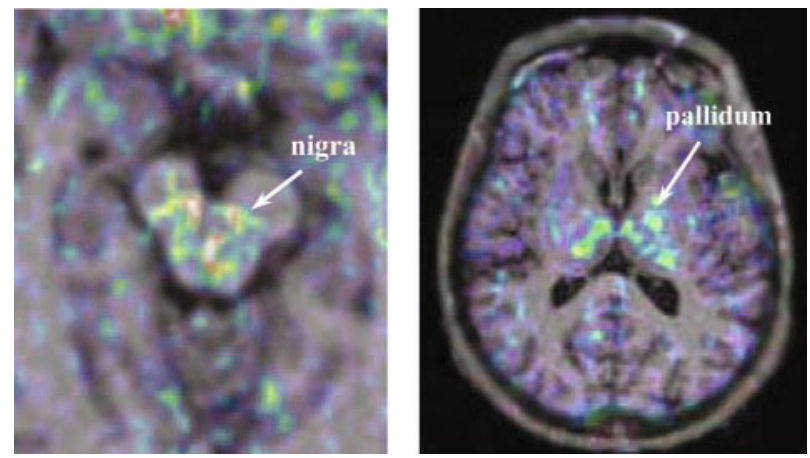

FIG. 2. Increased nigral and pallidal microglial activation in substantia nigra and pallidum, revealed by ${ }^{11} \mathrm{C}-\mathrm{PK} 11195$ PET in PD (picture courtesy of A Gerhard, MRC Clinical Sciences Center and Division of Neuroscience, Faculty of Medicine, Imperial College London, UK).

\section{MONITORING PD PROGRESSION}

There are a number of difficulties that arise when attempting to assess the progression of PD clinically. Disability rating scales are subjective, non-linear across grades, consider multiple aspects of the disorder, and are often biased toward particular symptoms. Additionally, within a year of diagnosis the majority of PD patients will require symptomatic therapy and this effectively masks disease progression. Attempts to wash out such medication usually achieve only partial success due to poor patient tolerance. It is now clear that for patients treated over several months a 2 -week washout of medication is insufficient to represent an unmedicated state.

PET and SPECT potentially provide potential biological markers for objectively monitoring disease progression in vivo in PD. In a limited series, striatal ${ }^{18} \mathrm{~F}$-dopa uptake has been shown to correlate with subsequent postmortem dopaminergic cell densities in the substantia nigra and striatal dopamine levels of patients ${ }^{41}$ and also of MPTP-lesioned monkeys. ${ }^{42}{ }^{18} \mathrm{~F}$-dopa PET is also highly reproducible ${ }^{43}$ and, at least in subjects with an intact dopamine system, appears to be uninfluenced both acutely ${ }^{44}$ and chronically ${ }^{45}$ by dopaminergic medication (see below). Although ${ }^{18} \mathrm{~F}$-dopa PET can be used as a marker of dopamine terminal function in PD, it may overestimate terminal density due to a relative upregulation of DDC in remaining neurons as a response to nigral cell loss. Given this, reductions in putamen ${ }^{18} \mathrm{~F}$-dopa will reflect both a failure of compensatory mechanisms along with nigral cell loss. Striatal ${ }^{123}$ I- $\beta$-CIT uptake has been shown to be unaffected by several weeks of exposure to L-dopa and dopamine agonists in PD patients. ${ }^{46}$ In contrast to ${ }^{18} \mathrm{~F}$-dopa, ${ }^{123} \mathrm{I}$ - $\beta$-CIT uptake may underestimate terminal density due to a relative downregulation of dopamine transporters in remaining neurons as a response to nigral cell loss.

Several series have demonstrated that loss of striatal ${ }^{18} \mathrm{~F}$-dopa uptake occurs more rapidly in PD than in age- matched controls. ${ }^{47-49}$ In early PD patients treated with L-dopa putamen, ${ }^{18} \mathrm{~F}$-dopa uptake has been reported to decline annually by around $10 \%$ of baseline (5\% of the normal mean), whereas caudate uptake falls at a slower rate. Similar rates of loss of putamen dopamine transporter binding have been reported with ${ }^{18} \mathrm{~F}-\mathrm{CFT} \mathrm{PET}^{50}$ and with ${ }^{123} \mathrm{I}-\beta$-CIT,${ }^{51}{ }^{123} \mathrm{I}$-FP-CIT, ${ }^{52}$ and ${ }^{123}$ I-IPT SPECT. ${ }^{53}$

\section{MODIFYING PD PROGRESSION}

As functional imaging can objectively follow loss of dopamine terminal function in $\mathrm{PD}$, it provides a potential means of monitoring the efficacy of putative neuroprotective agents. Dopamine agonists are one such possible class because they suppress endogenous dopamine production in vivo thus attenuating its oxidative metabolism and hence potential excessive hydroxyl free-radical formation. ${ }^{54}$ They are also weak antioxidants and free-radical scavengers, and some act as mitochondrial membrane stabilizers inhibiting caspase activation and the apoptotic cascade. Two different trials have examined the relative rates of loss of dopamine terminal function in early PD patients randomized to either a dopamine agonist or levodopa.

In the Ropinirole in Early Parkinson's Disease vs. L-dopa (REAL) PET 2-year double-blind multinational trial, 186 de novo PD patients were randomized (1:1) to either ropinirole or L-dopa. ${ }^{55}$ The primary endpoint was relative loss of putamen ${ }^{18} \mathrm{~F}$-dopa uptake, measured as an influx constant $\mathrm{Ki}$ while subjects were receiving medication. Scan data from six PET centers were transformed into standard stereotaxic space using an ${ }^{18} \mathrm{~F}$-dopa template to normalize brain position and shape. Parametric $\mathrm{Ki}$ images were then generated in standard space and sampled with a fixed region-of-interest (ROI) template. $\mathrm{Ki}$ images were also interrogated with statistical parametric mapping (SPM99) to localize voxel clusters in which significant between-group differences in rates of loss of dopaminergic function were occurring. Seventyfour percent of the ropinirole and $73 \%$ of the L-dopa group completed the study.

By the end of 2 years the cohorts were taking average daily doses of $12 \mathrm{mg}$ ropinirole or $560 \mathrm{mg}$ L-dopa. Fourteen percent of the ropinirole and $8 \%$ of the L-dopa group required open supplementary L-dopa. On blinded review, before statistical analysis, $11 \%$ of the untreated patients thought to have PD on clinical grounds were found to have normal caudate and putamen ${ }^{18} \mathrm{~F}$-dopa uptake at entry and this remained normal over the 2 years despite exposure to medication. As the trial primary endpoint was relative change in putamen ${ }^{18} \mathrm{~F}$-dopa this "normal" group was considered separately.

ROI analysis found that in subjects with both clinical and imaging evidence of PD, loss of putamen Ki was significantly slower over 2 years with ropinirole 
putamen peak voxel $\%$ change in $\mathrm{Ki}$

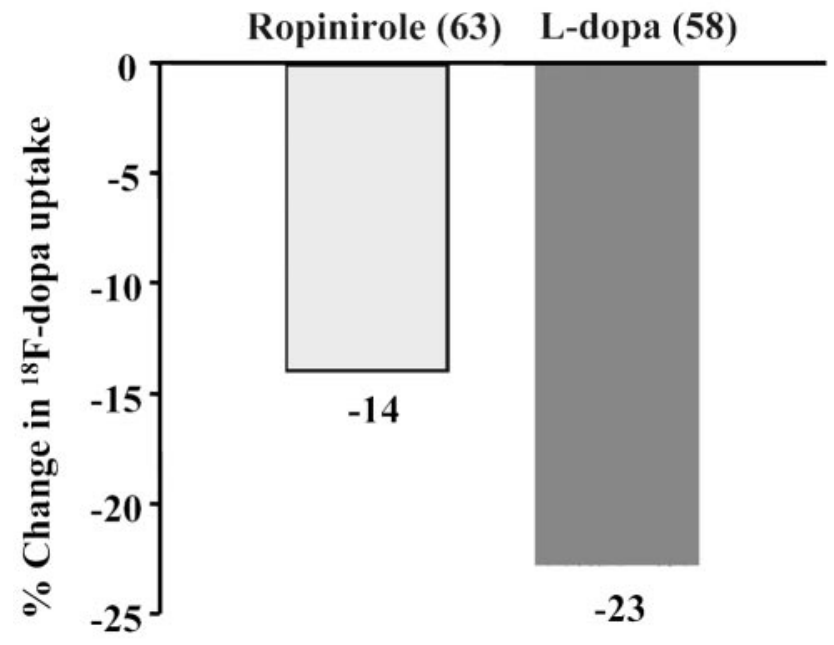

nigra peak voxel

$\%$ change in $\mathrm{Ki}$

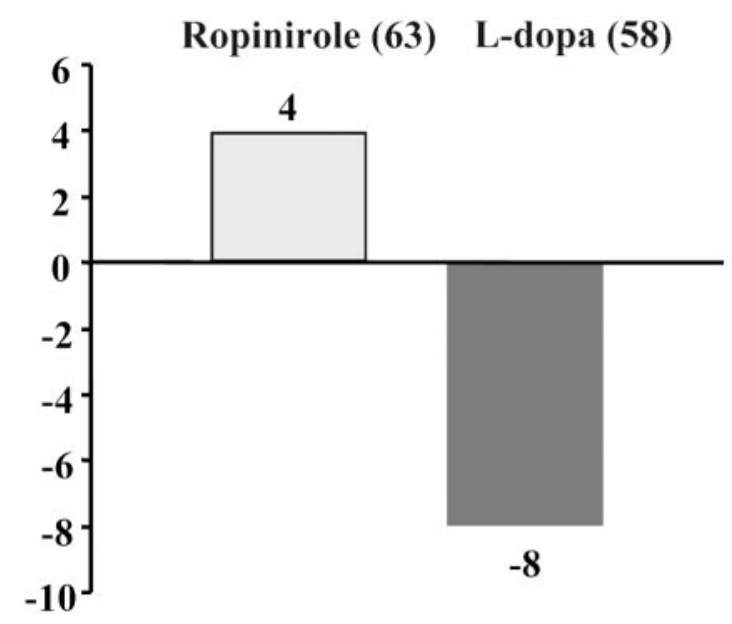

Relative $38 \%$ difference

$$
p<0.0001
$$

$p<0.025$

FIG. 3. SPM findings in the REAL-PET trial. Significant 2-year reductions in putamen and nigral ${ }^{18} \mathrm{~F}$-dopa uptake localized by SPM in cohorts of PD patients receiving either ropinirole or levodopa.

$(-13.4 \%)$ than with L-dopa $(-20.3 \% ; p=0.022)$. Statistical parametric mapping (SPM) confirmed this finding (putamen: ropinirole $-14.1 \%$; $-22.9 \%$ L-dopa; $p<$ 0.001) but also revealed that Ki reduction was significantly slower in substantia nigra taking ropinirole (substantia nigra: ropinirole $+4.3 \%$; L-dopa $-7.5 \%$; $p=$ 0.025) (FIG. 3). Clinically, the incidence of dyskinesia over 2 years was $27 \%$ with L-dopa but only $3 \%$ with ropinirole $(p<0.001)$. Improvements in mean Unified Parkinson's Disease Rating Scale (UPDRS) motor scores rated while taking medication were, however, superior by six points for the L-dopa cohort.

The second CALM-CIT trial involved a subgroup of 82 early PD patients from the CALM-PD study. ${ }^{56}$ This cohort was randomized 1:1 to the dopamine agonist pramipexole ( $0.5 \mathrm{mg} \mathrm{tds})$ or levodopa (100 $\mathrm{mg} \mathrm{tds})$ and had serial ${ }^{123}$ I- $\beta$-CIT SPECT over a 4 -year period. Open supplementary L-dopa was allowed if there was lack of therapeutic effect. Patients treated initially with pramipexole $(n=42)$ showed a significantly slower mean relative decline of striatal $\beta$-CIT uptake compared to subjects treated initially with levodopa $(n=40)$ at two $(47 \%)$, three (44\%), and four (37\%) years. Again, the incidence of complications was significantly reduced in the pramipexole cohort but improvement in UPDRS score, rated while taking medication, was greater in the L-dopa cohort.

These two trials therefore produced parallel findings, both suggesting that treatment with an agonist in early PD slows loss of dopamine terminal function by around one-third and delays treatment-associated complications. Concerns, however, have been raised concerning this interpretation. ${ }^{57}$ Two relevant issues are: 1) Could there have been ascertainment bias? Did more severely affected cases drop out of the agonist than the L-dopa arms due to lower efficacy of the former thus biasing the imaging changes in favor of agonist therapy? and 2) Can direct pharmacological effects of treatments on imaging parameters be excluded?

The fear of ascertainment bias appears to be unfounded in these trials. Patients recruited into the agonist and L-dopa arms had similar mean UPDRS scores off medication at entry and, when assessed taking medication at last visit, drop-outs showed no differential degree of disability. The issue concerning a direct and differential pharmacological effect of agonists and L-dopa on imaging, however, is more difficult. Animal studies have provided inconsistent data regarding $\beta$-CIT but highdose neuroleptic and apomorphine exposure have been shown to upregulate and downregulate DDC activity, respectively, in rodents. ${ }^{58}$ In the REAL PET trial the 19 cases with normal ${ }^{18} \mathrm{~F}$-dopa PET (14 taking L-dopa and five taking ropinirole) who were followed serially for 2 years continued to have normal findings with no significant changes in their mean $\mathrm{Ki}$ values. Patients with restless leg syndrome exposed to L-dopa or agonists for 
several months also have normal ${ }^{18} \mathrm{~F}$-dopa $\mathrm{PET}^{45}$ (Pavese and Brooks, unpublished data). In the CALM PD study there was a wash-in phase at 10 weeks involving $13 \mathrm{PD}$ patients and again, no significant effect of treatment on striatal $\beta$-CIT uptake was noted. In the ELLDOPA trial (see below), ${ }^{59} 22$ subjects entered as early cases of PD were found to have normal beta-CIT SPECT and this remained normal after exposure to daily doses of L-dopa varying from 150-600 mg over 9 months. These are, however, small groups of subjects and one cannot, therefore, rule out either a subtle imaging change due to direct effects of medication or a differential direct effect of agonists and L-dopa on a compromised dopamine system when administered long term.

The finding of dissociated imaging and clinical outcomes raises the question as to what is the appropriate clinical outcome for comparison. Patients were rated with the UPDRS when taking medication and so this could have masked true disease status. Additionally, over a 2-year period in the early honeymoon phase little clinical progression is normally seen. What is clear is that the prevalence of complications was far higher in the L-dopa arms of both the REAL PET and CALM-PD trials correlating with the more rapid progression detected with imaging. The real test, of course, will be whether early use of agonists delays the need for institutional care in the longer term.

The REAL PET and CALM-CIT trials compared two active medications without including a placebo group. In the ELLDOPA study, 135 early PD patients were randomized to treatment with $150 \mathrm{mg}, 300 \mathrm{mg}$, or $600 \mathrm{mg}$ L-dopa or placebo for 40 weeks. Loss of striatal ${ }^{123} \mathrm{I}-\beta$ CIT uptake was significantly greater in the group treated with $600 \mathrm{mg}$ levodopa compared to placebo if subjects with normal imaging were excluded. This study, though limited by its short duration and small sample size, suggests that L-dopa treatment results in greater loss of striatal ${ }^{123}$ I- $\beta$-CIT uptake compared to placebo.

\section{RESTORATIVE APPROACHES IN PARKINSON'S DISEASE}

\section{Human fetal cell implantation trials}

As well as providing a means of following natural disease progression and monitoring the effects of putative neuroprotective agents, functional imaging provides a means of examining the efficacy of restorative approaches to PD. Possible approaches include: striatal implants of human and porcine fetal mesencephalic cells, retinal cells that release levodopa/dopamine, and transformed cells which secrete dopamine, nerve growth factors, or express antiapoptotic genes, neural progenitor cells, and direct intrastriatal infusions of nerve growth factors.
There have now been several open series detailing clinical and ${ }^{18} \mathrm{~F}$-dopa PET findings in advanced PD patients after implantation of fetal mesencephalic cells or tissue into striatum. ${ }^{60}$ The Lund group have reported serial clinical and ${ }^{18} \mathrm{~F}$-dopa PET findings over a period of 10 years on two PD patients following implantation of fetal midbrain cells into the putamen contralateral to their more affected limbs. ${ }^{61}$ Both of these patients have maintained a clinical improvement, particularly in "on" time which went from $40 \%$ to all of the day in one unilaterally grafted case whose ${ }^{18} \mathrm{~F}$-dopa uptake into the implanted putamen reached the lower end of the normal range. It was subsequently demonstrated that the graft released normal synaptic levels of dopamine after an amphetamine challenge.

Another four unilaterally transplanted PD patients showed ${ }^{18}$ F-dopa PET evidence of graft function 1 year following surgery and three responded clinically to transplantation but the fourth deteriorated and now has signs suggestive of multiple system atrophy. ${ }^{62}$ In a subsequent series of four bilaterally transplanted patients a mean $50 \%$ improvement in UPDRS scores was demonstrated at 2 years associated with a $60 \%$ increase in putamen and $30 \%$ increase in caudate ${ }^{18} \mathrm{~F}$-dopa uptake. ${ }^{63}$

Clinically successful transplantation of fetal tissue with corroborative serial ${ }^{18} \mathrm{~F}$-dopa PET findings has also been reported for five PD patients in a 2-year open follow-up French study ${ }^{64}$ and for six PD patients in a 2-year follow-up series from Tampa, Florida. ${ }^{65}$ In the French study grafted putamen Ki values correlated with percentage time "on" during the day and finger dexterity while in an "off" state. Two of the transplanted PD patients in the Florida series died from unrelated causes and at postmortem viable tyrosine hydroxylase staining graft tissue, forming connections with host neurons, was found. ${ }^{66}$

Given the encouraging findings of these pilot open series, two major double-blind controlled trials on the efficacy of implantation of human fetal cells in PD were sponsored by the National Institutes of Health (NIH) in the United States. The first of these involved 40 patients who were 34-75 years of age and had severe Parkinson's disease (mean duration, 14 years). ${ }^{67}$ These were randomized to receive either an implant of human fetal mesencephalic tissue or to undergo sham surgery and were followed for 1 year with a subsequent extension to 3 years. In the transplant recipients, mesencephalic tissue from four embryos cultured for up to 1 month was implanted into the putamen bilaterally (two embryos per side) via a frontal approach. In the patients who underwent sham surgery, holes were drilled in the skull but the dura was not penetrated. No immunotherapy was used. The transplanted patients showed no significant improvement in the primary endpoint, clinical global impression, 
at 1 year but there was a significant mean $18 \%$ improvement in the mean UPDRS motor score compared with the sham-surgery group when tested in the morning before receiving medication $(p=0.04)$. This improvement was more evident for patients under 60 years of age (34\% improvement, $p=0.005$ ). At 3 years mean total UPDRS score was improved $38 \%$ in the younger and $14 \%$ in the older transplanted groups (both $p<0.01$ ). Sixteen of 19 transplanted patients showed an increase in putamen ${ }^{18} \mathrm{~F}$ dopa uptake (group mean increase $40 \%$ ) and increases were similar in the younger and older cohorts. ${ }^{70}$ A problem was that "off" dystonia and dyskinesias developed in $15 \%$ of the patients who received transplants in this series, even after reduction of levodopa.

More recently the second NIH trial was reported. ${ }^{68}$ Here, 34 patients were randomized to receive bilateral implants of fetal mesencephalic tissue from four fetuses or one fetus per side into posterior putamen or had sham surgery (a partial burr hole without penetration of the dura). Fetal tissue was cultured for less than $48 \mathrm{~h}$ before transplantation and all patients received immunosuppression for 6 months after surgery. The trial duration was 2 years and the primary outcome variable was change in UPDRS motor score. 31 patients completed and two patients died during the trial, whereas another three died subsequently from unrelated causes. At postmortem the transplanted patients showed significantly higher tyrosine hydroxylase staining in the putamen relative to the sham-grafted treated patients with graft innervation evident. Mean putamen:occipital ${ }^{18} \mathrm{~F}$-dopa uptake ratios were unchanged in the control patients but showed $20 \%$ and $30 \%$ increases in patients receiving tissue from one and four fetuses, respectively. The mean UPDRS motor score off medication for the controls deteriorated by 9.4 and 3.5 points over 2 years for the control group and one fetus group and improved by 0.7 points for the four fetus groups. These clinical changes were not significantly different overall (four fetus $v s$ controls $p=0.096$ ) but less severely affected cases were significantly improved $(p=0.006)$. Additionally, no significant differences in "on" time without dyskinesias, total "off" time, activities of daily living (ADL) scores, or levodopa dose required were evident between the groups. "Off" period dyskinesias were evident in 13 of 23 implanted patients but were not seen in the control arm.

In summary, despite both histological and ${ }^{18} \mathrm{~F}$-dopa PET evidence of graft function, neither of these controlled trials demonstrated clinical efficacy with their primary endpoints and in both trials "off" period dyskinesias were problematic. There were indications, however, that younger, less severely affected patients could benefit from intrastriatal implantation of human fetal dopamine cells.

\section{Intraputaminal GDNF infusions}

GDNF is a potent neurotrophic factor known to protect dopamine neurons in rodents and nonhuman primates against toxins such as 6-hydroxydopamine or MPTP. The safety and efficacy of infusing GDNF directly into the posterior putamen has been recently studied in a small open trial. ${ }^{69}$ Five PD patients had in-dwelling catheters inserted and have tolerated continuous glial-derived neurotrophic factor (GDNF) delivery at levels of 14-40 $\mu \mathrm{g} /$ day $(6 \mu \mathrm{l} /$ hour $)$ for over 2 years, unilaterally in one patient and bilaterally in four patients, without serious side effects. Significant improvements at 12 months were reported in UPDRS subscores: $39 \%$ and $61 \%$ improvements in the off-medication motor III and ADL II subscales, respectively. No change in cognitive status was detected on a battery of behavioral tests. Regions of interest sited in the vicinity of the catheter tip showed $18-24 \%$ increases in putaminal ${ }^{18} \mathrm{~F}$-dopa $\mathrm{Ki}$ which were confirmed with statistical parametric mapping. Additionally, SPM detected 16-26\% increases in nigral dopamine storage at 6 months, suggesting that retrograde transport of GDNF may have occurred. These findings imply that putamen GDNF infusion is safe and may represent a potential restorative approach for $\mathrm{PD}$.

\section{FLUCTUATIONS AND DYSKINESIAS}

PD patients with fluctuating responses to levodopa show lower putamen ${ }^{18} \mathrm{~F}$-dopa uptake than those with early disease and sustained therapeutic responses. ${ }^{70} \mathrm{~A}$ confounder, however, when trying to compare pre-synaptic dopamine terminal function in these groups is that the former tend to have an earlier age of disease onset, more severe disease, longer disease duration, and a greater cumulative exposure to levodopa. By using analysis of covariance to factor out effects of age at onset and disease duration in groups of fluctuators and nonfluctuators and also by matching subgroups of these patients for age of onset and disease duration, De La Fuente Fernandez and colleagues ${ }^{70}$ concluded that mean putamen ${ }^{18} \mathrm{~F}$ dopa uptake was $28 \%$ lower in PD patients with motor complications compared with those without complications but that there was considerable overlap of the two individual ranges. Although loss of putamen dopamine terminal function predisposes PD patients to development of levodopa-associated complications, it cannot be solely responsible for determining the timing of onset of fluctuations and involuntary movements. As mentioned earlier, another factor may be loss of pallidal dopamine storage capacity which is initially upregulated and then later falls below normal. ${ }^{18}$

Dopamine receptors fall into D1 (D1, D5) and D2 (D2, D3, D4) subclasses. The striatum contains mainly D1 and D2 receptor subtypes and these both play a role in modulating locomotor function. PET with spiperone- 
based tracers and ${ }^{123}$ I-IBZM SPECT studies have reported normal levels of striatal D2-binding in untreated $\mathrm{PD}$, whereas ${ }^{11} \mathrm{C}$-raclopride PET has detected $10-20 \%$ increases in putamen D2 site availability. ${ }^{71,72}$ In PD chronically exposed to levodopa, both ${ }^{11} \mathrm{C}$-methylspiperone PET and ${ }^{123}$ I-IBZM SPECT studies have reported normal or mildly reduced striatal D2 binding. ${ }^{71}$ Serial ${ }^{11} \mathrm{C}$-raclopride PET has shown that after 6 months of exposure to levodopa the mildly increased putamen ${ }^{11} \mathrm{C}$ raclopride binding seen in de novo $\mathrm{PD}$ patients subsequently normalizes. ${ }^{73}$ Chronically levodopa-exposed PD cases continue to show normal levels of putamen D2 binding, explaining their good locomotor response to levodopa. ${ }^{74}{ }^{11} \mathrm{C}-\mathrm{SCH} 23390$ PET, a marker of D1 site binding, has revealed normal striatal uptake in de novo $\mathrm{PD},{ }^{74}$ whereas patients who had been exposed to levodopa for several years showed a $20 \%$ reduction in striatal binding. These findings are in good agreement with in vitro reports of striatal dopamine D1 and D2 receptor binding based on postmortem material from end-stage patients.

Striatal dopamine D1 and D2 receptor availability has been examined in subgroups of dyskinetic and non-dyskinetic PD patients matched for clinical disease duration, disease severity, and daily levodopa dosage ${ }^{74}$ Mean caudate and putamen D1 and putamen D2 binding were normal in both the dyskinetic and the nondyskinetic PD subgroups, whereas caudate D2 binding was reduced in each by around $15 \%$.

Other series have also reported similar striatal dopamine D1 and D2 binding in fluctuating and dyskinetic PD patients compared with sustained responders. ${ }^{75}$ These findings, therefore, suggest that onset of motor complications in PD is not primarily associated with alterations in striatal dopamine receptor availability.

${ }^{11} \mathrm{C}$-raclopride competes with endogenous synaptic dopamine when binding to D2 receptors. ${ }^{76}{ }^{11} \mathrm{C}$-raclopride PET, therefore, allows one indirectly to monitor changes in levels of striatal dopamine release. When early nonfluctuating PD patients are given $3 \mathrm{mg} / \mathrm{kg}$ of levodopa as an intravenous bolus they show a mean $10 \%$ fall in posterior putamen ${ }^{11} \mathrm{C}$-raclopride binding while advanced cases with fluctuations show a $23 \%$ fall. ${ }^{77}$ These reductions in putamen ${ }^{11} \mathrm{C}$-raclopride binding correlate with disease severity assessed with the UPDRS when off medication and indicate that, as loss of dopamine terminals in PD increases, striatal buffering of dopamine fails when exogenous levodopa is administered. This failure reflects a combination of upregulation of striatal dopamine synthesis and release from levodopa by the remaining terminals along with severe loss of dopamine transporters, preventing reuptake.

De la Fuente-Fernandez and colleagues ${ }^{78}$ have examined changes in ${ }^{11} \mathrm{C}$-raclopride binding after an oral levodopa challenge in early and advanced PD. They reported that patients with a sustained response to levodopa had an estimated two-fold rise in synaptic dopamine levels at 1 hour after drug administration which increased to ninefold by 4 hours. In contrast, fluctuating responders increased their synaptic dopamine levels six-fold by 1 hour, explaining their more rapid response to medication, but showed baseline dopamine levels at 4 hours. It was also noted that "off" episodes after levodopa did not necessarily coincide with low synaptic dopamine levels.

These findings suggest that fluctuating responders develop more pulsatile swings in their synaptic dopamine levels after oral levodopa due to a failure of their remaining terminals to buffer exogenous dopamine. When dopamine binds to postsynaptic receptors they become transiently internalized and thus unavailable. Normal tonic release of dopamine maintains a steady population of available receptors on the cell surface but pathologically pulsatile swings may cause inappropriately high levels of internalization, leading to refractory neurons and unpredictable "offs."

Medium spiny projection neurons in the caudate and putamen release endogenous opioids and substance $P$ along with GABA into external and internal pallidum. ${ }^{79}$ Striatal projections to external pallidum (GPe) contain enkephalin which binds mainly to $\delta$-opioid sites and inhibits GABA release in the GPe. Striatal projections to GPi transmit dynorphin, which binds to $\kappa$-opioid sites and inhibits glutamate release from subthalamic afferents to the GPi, and also substance P (SP), which binds to neurokinin NK1 receptors. It is thought that phasic burst firing of striatopallidal projection neurons results primarily in GABA release, whereas sustained tonic firing causes additional modulatory opioid and SP release. The caudate and putamen also contain high densities of $\mu$-opioid, $\kappa$-opioid, and $\delta$-opioid sites. These receptors are located both presynaptically on dopamine terminals in which they regulate dopamine release and postsynaptically on interneurons and medium spiny projection neurons to pallidum terminals.

There is now strong evidence supporting the presence of deranged opioid and SP transmission in the basal ganglia of PD patients both from postmortem studies and lesion animal models of this disorder. ${ }^{80-82}$ At postmortem, end-stage treated PD patients show raised levels of pallidal preproenkephalin. In rats lesioned with the nigral toxin 6-OHDA there are raised levels of striatal enkephalin and preproenkephalin expression, whereas prodynorphin expression is suppressed. When such animals are made hyperkinetic or frankly dyskinetic after chronic exposure to pulsatile doses of levodopa, further overexpression of striatal preproenkephalin is seen along with raised expression of prodynorphin and SP. Levodopanaïve MPTP-lesioned monkeys have also been reported to show raised striatal enkephalin and reduced SP mRNA expression. Exposure to levodopa for 1 month failed to 


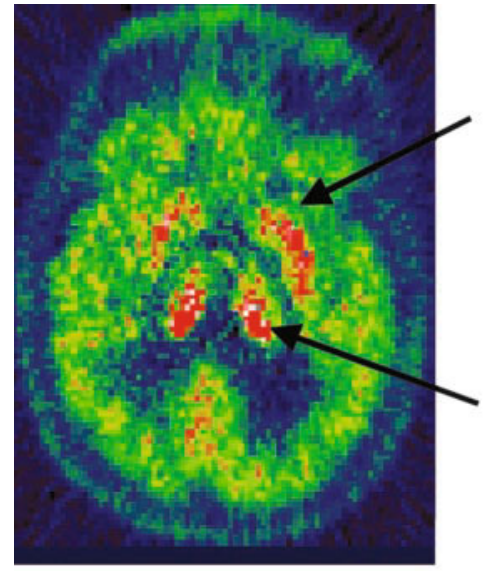

PD

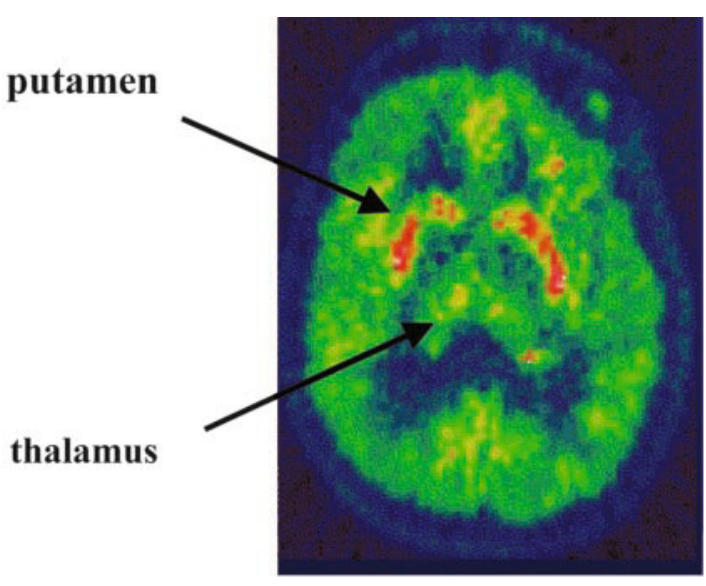

PD + dyskinesias

FIG. 4. Striatal and thalamic NK1 binding of ${ }^{18} \mathrm{~F}-\mathrm{L} 829165$ in nondyskinetic and dyskinetic PD patients. Thalamic signal is reduced in the latter (picture courtesy of A. Whone, MRC Clinical Sciences Center and Division of Neuroscience, Faculty of Medicine, Imperial College London, UK).

normalize striatal preproenkephalin mRNA expression, whereas SP mRNA expression became elevated.

${ }^{11} \mathrm{C}$-diprenorphine PET is a nonselective marker of $\mu$-opioid, $\kappa$-opioid, and $\delta$-opioid sites and its binding is sensitive to levels of endogenous opioids. ${ }^{18} \mathrm{~F}-\mathrm{L} 829165$ PET is a selective marker of NK1 site availability. If raised basal ganglia levels of enkephalin, dynorphin, and SP are associated with levodopa-induced dyskinesias (LIDs), PD patients with motor complications could be expected to show reduced binding of ${ }^{11} \mathrm{C}$-diprenorphine and ${ }^{18} \mathrm{~F}$-L829165 compared with those with sustained treatment responses. Piccini and coworkers ${ }^{83}$ have reported significant reductions in ${ }^{11} \mathrm{C}$-diprenorphine binding in caudate, putamen, thalamus, and anterior cingulate in dyskinetic patients compared with sustained responders. Individual levels of putamen ${ }^{11} \mathrm{C}$-diprenorphine uptake correlated inversely with severity of dyskinesia. In a preliminary study, thalamic NK1 availability has been shown to be reduced in dyskinetic PD patients but normal in nondyskinetic cases (FIG. 4). ${ }^{84}$ These in vivo findings support the presence of elevated levels of endogenous peptides in the basal ganglia of dyskinetic PD patients and suggest that this, rather than a primary alteration in dopamine receptor availability, may in part responsible for the appearance of involuntary movements.

\section{DEMENTIA AND PD}

The prevalence of dementia is raised two-fold in PD and possible causes include direct cortical involvement by diffuse Lewy body (DLB) disease, coincident Alzheimer's disease (AD), small vessel disease, and loss of cholinergic projections. ${ }^{85}$ In addition, DLB is thought to be the pathological diagnosis in around $20 \%$ of cases with the clinical picture of AD.

PET studies have shown increased levels of resting oxygen and glucose metabolism in the contralateral lentiform nucleus of hemiparkinsonian patients with early disease, whereas PD patients with established bilateral involvement have normal levels of lentiform metabolism. ${ }^{86,87}$ Covariance analysis reveals an abnormal profile of relatively raised resting lentiform nucleus and lowered frontal metabolism in PD patients with established disease, even when absolute values are normal. ${ }^{88}$ The degree of expression of this profile correlates with clinical disease severity. ${ }^{89}$

Nondemented PD patients show normal cortical metabolism, whereas 18F-2-fluoro-2-deoxyglucose $\left({ }^{18} \mathrm{FDG}\right)$ PET scans of frankly demented PD patients show an Alzheimer pattern of impaired brain glucose utilization, with posterior parietal and temporal association areas being most affected. ${ }^{90,91}$ Currently, it remains unclear whether the pattern of glucose hypometabolism in demented PD patients reflects coincidental AD, cortical Lewy body disease, loss of cholinergic projections, or some other degenerative process. Clinicopathological series suggest that there is considerable overlap in the cortical FDG PET findings of coincidental AD and cortical Lewy body disease but that cortical Lewy body disease cases show a greater reduction in resting glucose metabolism of the primary visual cortex. ${ }^{92}$ Interestingly, in one series one-third of nondemented PD patients with established disease showed subclinical resting temporoparietal cortical metabolic dysfunction with both ${ }^{18}$ FDG PET and phosphorus nuclear magnetic resonance $\left({ }^{31} \mathrm{P}-\mathrm{NMR}\right)$ spectroscopy. ${ }^{93}$ Whether these are cases that will go on to become demented is still unclear.

Whether DLB and PD represent opposite ends of a spectrum is unclear but DLB patients show not only cerebral cortical neuronal loss, with Lewy bodies in surviving neurones, but also loss of nigrostriatal dopami- 
nergic neurons. In contrast, nigral pathology is mild in AD. Using ${ }^{123}$ I-FP-CIT SPECT, Walker and colleagues ${ }^{94}$ examined striatal DAT binding in 27 patients with clinically presumed DLB, 17 with AD, 19 drugnaive patients with $\mathrm{PD}$, and 16 controls. The presumed DLB and PD patients had significantly lower uptake of caudate and putamen ${ }^{123}$ I-FP-CIT than patients with AD $(p<0.001)$ and controls $(p<0.001)$. A problem, however, with the interpretation of this finding is that the DLB and PD patient groups had equivalent parkinsonism on their UPDRS and Hoehn and Yahr ratings, whereas the AD patients and controls had slight or no rigidity. The SPECT findings could therefore simply have reflected selection bias on the basis of rigidity. The authors, however, were subsequently able to correlate their SPECT findings with 10 postmortem examinations. Nine of these 10 cases were thought to have DLB in life but only four had this diagnosis at autopsy. All four had reduced striatal ${ }^{123}$ I-FP-CIT uptake. Five of the 10 cases had AD pathology and four of the five had normal ${ }^{123} \mathrm{I}-$ FP-CIT SPECT. These clinicoimaging correlations suggest that ${ }^{123}$ I-FP-CIT SPECT may be helpful in discriminating DLB from $\mathrm{AD}$ but its role in patients with isolated dementia without parkinsonism remains unproven.

\section{CONCLUSIONS}

In summary, functional imaging studies can offer an objective method of assessing disease progression in PD thereby avoiding problems associated with trials of putative neuroprotective agents that have relied on clinical assessment alone. PET and SPECT studies, however, provide markers of nerve terminal function rather than cell density and thus, like clinical assessments, are potentially influenced by compensatory mechanisms and direct effects of medication. In addition, PET can monitor the glial activation associated with PD and potentially determine the anti-inflammatory properties of putative neuroprotective agents. Finally, imaging findings suggest that the fluctuating responses to levodopa experienced by advanced PD cases are a consequence of loss of pallidal along with putamen dopamine storage capacity and pathological swings in synaptic dopamine levels, whereas dyskinesias are associated with excessive levels of opioid peptide and SP transmission.

\section{REFERENCES}

1. Hu MT, White SJ, Herlihy AH et al. A comparison of (18)F-dopa PET and inversion recovery MRI in the diagnosis of Parkinson's disease. Neurology 56:1195-1200, 2001.

2. Hutchinson M, Raff U. Structural changes of the substantia nigra in Parkinson's disease as revealed by MR imaging. AJNR Am J Neuroradiol 21:697-701, 2000.

3. Schrag A, Good CD, Miszkiel K et al. Differentiation of atypical parkinsonian syndromes with routine MRI. Neurology 54:697702,2000 .
4. Schulz JB, Skalej M, Wedekind D et al. Magnetic resonance imaging-based volumetry differentiates idiopathic Parkinson's syndrome from multiple system atrophy and progressive supranuclear palsy. Ann Neurol 45:65-74, 1999.

5. Seppi K, Schocke MF, Esterhammer R et al. Diffusion-weighted imaging discriminates progressive supranuclear palsy from PD, but not from the parkinson variant of multiple system atrophy. Neurology 60:922-927, 2003

6. Jellinger K. The pathology of parkinsonism. In: Movement disorders 2 (Marsden CD, Fahn S, eds), pp 124-165. Ed 1. London: Butterworths, 1987.

7. Fearnley JM, Lees AJ. Ageing and Parkinson's disease: substantia nigra regional selectivity. Brain 114:2283-2301, 1991.

8. Kish SJ, Shannak K, Hornykiewicz O. Uneven pattern of dopamine loss in the striatum of patients with idiopathic Parkinson's disease. N Engl J Med 318:876-880, 1988.

9. Braak H, Tredici KD, Rub U et al. Staging of brain pathology related to sporadic Parkinson's disease. Neurobiol Aging 24:197211, 2003.

10. Jellinger KA, Seppi K, Wenning GK, Poewe W. Impact of coexistent Alzheimer pathology on the natural history of Parkinson's disease. J Neural Transm 109:329-339, 2002.

11. McKeith IG, Galasko D, Kosaka K et al. Consensus guidelines for the clinical and pathologic diagnosis of dementia with Lewy bodies (DLB): report of the consortium on DLB international workshop. Neurology 47:1113-1124, 1996.

12. Morrish PK, Sawle GV, Brooks DJ. Clinical and [18F]dopa PET findings in early Parkinson's disease. J Neurol Neurosurg Psychiatry 59:597-600, 1995.

13. Rakshi JS, Uema T, Ito $\mathrm{K}$ et al. Frontal, striatal, and midbrain dopaminergic function in early and advanced Parkinson's disease: a 3D 18F-dopa PET study. Brain 122:1637-1650, 1999.

14. Brooks DJ, Ibañez V, Sawle GV et al. Differing patterns of striatal 18 F-dopa uptake in Parkinson's disease, multiple system atrophy and progressive supranuclear palsy. Ann Neurol 28:547-555, 1990.

15. Leenders KL, Salmon EP, Tyrrell P et al. The nigrostriatal dopaminergic system assessed in vivo by positron emission tomography in healthy volunteer subjects and patients with Parkinson's disease. Arch Neurol 47:1290-1298, 1990.

16. Bernheimer H, Birkmayer W, Hornykiewicz O et al. Brain dopamine and the syndromes of Parkinson and Huntington. Clinical, morphological, and neurochemical correlations. J Neurol Sci 20: $415-455,1973$.

17. Lindvall O, Bjorklund A. Dopaminergic innervation of the globus pallidus by collaterals from the nigrostriatal pathway. Brain Res 172:169-173, 1979.

18. Whone AL, Moore RY, Piccini P, Brooks DJ. Plasticity in the nigropallidal pathway in Parkinson's disease: an 18F-dopa PET study. Ann Neurol 53:206-213, 2003.

19. Frost JJ, Rosier AJ, Reich SG et al. Positron emission tomographic imaging of the dopamine transporter with 11C-WIN 35,428 reveals marked declines in mild Parkinson's disease. Ann Neurol 34:423431, 1993.

20. Rinne JO, Bergman J, Ruotinnen $\mathrm{H}$ et al. Striatal uptake of a novel PET ligand, [18F]b-CFT, is reduced in early Parkinson's disease. Synapse 31:119-124, 1999.

21. Guttman M, Burkholder J, Kish SJ et al. [11C]RTI-32 PET studies of the dopamine transporter in early dopa-naive Parkinson's disease: implications for the symptomatic threshold. Neurology 48: 1578-1583, 1997.

22. Marek K, Seibyl JP, Zoghbi SS et al. [I-123] $\beta$-CIT SPECT imaging demonstrates bilateral loss of dopamine transporters in hemiparkinsons disease. Neurology 46:231-237, 1996.

23. Benamer HTS, Patterson J, Wyper DJ et al. Correlation of Parkinson's disease severity and duration with I-123-FP-CIT SPECT striatal uptake. Movement Disorders 15:692-698, 2000.

24. Benamer TS, Patterson J, Grosset DG et al. Accurate differentiation of parkinsonism and essential tremor using visual assessment of [123I]-FP-CIT imaging: the [123I]-FP-CIT study group. Mov Disord 15:503-510, 2000.

25. Fischman AJ, Bonab AA, Babich JW et al. [C-11, I-127] Altropane: a highly selective ligand for PET imaging of dopamine transporter sites. Synapse 39:332-342, 2001. 
26. Mozley PD, Schneider JS, Acton PD et al. Binding of [Tc99m]TRODAT-1 to dopamine transporters in patients with Parkinson's disease and in healthy volunteers. J Nucl Med 41:584-589, 2000.

27. Vingerhoets FJG, Schulzer M, Caine DB, Snow BJ. Which clinical sign of Parkinson's disease best reflects the nigrostriatal lesion? Ann Neurol 41:58-64, 1997.

28. Otsuka M, Ichiya Y, Kuwabara Y et al. Differences in the reduced 18F-dopa uptakes of the caudate and the putamen in Parkinson's disease: correlations with the three main symptoms. J Neurol Sci 136:169-173, 1996.

29. Tatsch K, Schwarz J, Mozley PD et al. Relationship between clinical features of Parkinson's disease and presynaptic dopamine transporter binding assessed with [I-123]IPT and SPECT. Eur J Nucl Med 24:415-421, 1997.

30. Lee CS, Samii A, Sossi V et al. In vivo positron emission tomographic evidence for compensatory changes in presynaptic dopaminergic nerve terminals in Parkinson's disease. Ann Neurol 47: 493-503, 2000.

31. Doder M, Rabiner EA, Turjanski N et al. Tremor in Parkinson's disease and serotonergic dysfunction: an (11)C-WAY 100635 PET study. Neurology 60:601-605, 2003.

32. Golbe LI. The genetics of Parkinson's disease: a reconsideration. Neurology 40(Suppl 3):7-16, 1990.

33. Piccini P, Morrish PK, Turjanski N et al. Dopaminergic function in familial Parkinson's disease: a clinical and 18F-dopa PET study. Ann Neurol 41:222-229, 1997.

34. Piccini P, Burn DJ, Ceravalo R et al. The role of inheritance in sporadic Parkinson's disease: evidence from a longitudinal study of dopaminergic function in twins. Ann Neurol 45:577-582, 1999.

35. Khan NL, Brooks DJ, Pavese $\mathrm{N}$ et al. Progression of nigrostriatal dysfunction in a parkin kindred: an [18F]dopa PET and clinical study. Brain 125:2248-2256, 2002.

36. Hilker R, Klein C, Ghaemi M et al. Positron emission tomographic analysis of the nigrostriatal dopaminergic system in familial parkinsonism associated with mutations in the parkin gene. Ann Neurol 49:367-376, 2001

37. Scherfler C, Khan NL, Pavese N et al. Striatal and extrastriatal PET measurements of dopa metabolism and D2 receptor status in patients with parkin gene mutations. Neurology 58(Suppl 3):A201, 2002.

38. Banati RB, Myers R, Kreutzberg GW. PK ('peripheral benzodiazepine')-binding sites in the CNS indicate early and discrete brain lesions: microautoradiographic detection of [3H]PK11195 binding to activated microglia. J Neurocytol 26:77-82, 1997.

39. McGeer PL, Itagaki S, Boyes BE, McGeer EG. Reactive microglia are positive for HLA-DR in the substantia nigra of Parkinson's and Alzheimer's disease brains. Neurology 38:1285-1291, 1988.

40. Gerhard A, Banati RB, Cagnin A, Brooks DJ. In vivo imaging of activated microglia with [C-11]PK11195 positron emission tomography (PET) in idiopathic and atypical Parkinson's disease. Neurology 56(Suppl 3):A270, 2001.

41. Snow BJ, Tooyama I, McGeer EG et al. Human positron emission tomographic $[18 \mathrm{~F}]$ fluorodopa studies correlate with dopamine cell counts and levels. Ann Neurol 34:324-330, 1993.

42. Pate BD, Kawamata T, Yamada T et al. Correlation of striatal fluorodopa uptake in the MPTP monkey with dopaminergic indices. Ann Neurol 34:331-338, 1993.

43. Rakshi JS, Bailey DL, Morrish PK, Brooks DJ. Implementation of $3 \mathrm{D}$ acquisition, reconstruction, and analysis of dynamic [18F] Fluorodopa studies. In: Quantification of brain function using PET (Myers R, Cunningham V, Bailey D, Jones T, eds), pp 82-87. San Diego: Academic Press, 1996.

44. Ceravolo R, Piccini P, Bailey DL et al. 18F-dopa PET evidence that tolcapone acts as a central COMT inhibitor in Parkinson's disease. Synapse 43:201-207, 2002.

45. Turjanski N, Lees AJ, Brooks DJ. Striatal dopaminergic receptor dysfunction in patients with restless legs syndrome: 18F-dopa and 11C-raclopride PET studies. Neurology 52:932-937, 1999.

46. Innis RB, Marek KL, Sheff K et al. Effect of treatment with L-dopa/carbidopa or L-selegiline on striatal dopamine transporter SPECT imaging with [I-123] $\beta$-CIT. Mov Disord 14:436-442, 1999.
47. Vingerhoets FJG, Snow BJ, Lee CS et al. Longitudinal fluorodopa positron emission tomographic studies of the evolution of idiopathic parkinsonism. Ann Neurol 36:759-764, 1994.

48. Morrish PK, Rakshi JS, Sawle GV, Brooks DJ. Measuring the rate of progression and estimating the preclinical period of Parkinson's disease with [18F]dopa PET. J Neurol Neurosurg Psychiatry 64: $314-319,1998$.

49. Nurmi E, Ruottinen HM, Bergman $\mathrm{J}$ et al. Rate of progression in Parkinson's disease: a 6-[18F]fluoro-L-dopa PET study. Mov Disord 16:608-615, 2001.

50. Nurmi E, Ruottinen HM, Kaasinen V et al. Progression in Parkinson's disease: a positron emission tomography study with a dopamine transporter ligand. Ann Neurol 47:804-808, 2000.

51. Marek K, Innis R, van Dyck C et al. [123I] $\beta$-CIT SPECT imaging assessment of the rate of Parkinson's disease progression. Neurology 57:2089-2094, 2001.

52. Winogrodzka A, Bergmans P, Booij J et al. [123I]FP-CIT SPECT is a useful method to monitor the rate of dopaminergic degeneration in early-stage Parkinson's disease. J Neural Transm 108: 1011-1019, 2001.

53. Schwarz J, Tatsch K, Linke R et al. Measuring the decline of dopamine transporter binding in patients with Parkinson's disease using 123I-IPT and SPECT. Neurology 48(Suppl 2):A208, 1997.

54. Olanow CW, Jenner P, Brooks D. Dopamine agonists and neuroprotection in Parkinson's disease. Ann Neurol 44(Suppl1):S167S174, 1998.

55. Whone AL, Watts RL, Stoessl J et al. Slower progression of PD with ropinirol versus L-dopa: the REAL-PET study. Ann Neurol 54:93-101, 2003.

56. Parkinson Study Group. Dopamine transporter brain imaging to assess the effects of pramipexole vs levodopa Parkinson disease progression. JAMA 287:1653-1661, 2002.

57. Ahlskog JE. Slowing Parkinson's disease progression: recent dopamine agonist trials. Neurology 60:381-389, 2003.

58. Hadjiconstantinou M, Wemlinger TA, Sylvia CP et al. Aromatic L-amino acid decarboxylase activity of mouse striatum is modulated via dopamine receptors. J Neurochem 60:2175-2180, 1993.

59. Parkinson Study Group. Does levodopa slow or hasten the rate of progression of Parkinson disease? The results of the ELLDOPA trial. Neurology 60(Suppl 1):A80-81, 2003.

60. Lindvall O. Cerebral implantation in movement disorders: state of the art. Mov Disord 14:201-205, 1999.

61. Piccini P, Brooks DJ, Bjorklund A et al. Dopamine release from nigral transplants visualised in vivo in a Parkinson's patient. Nat Neurosci 2:1137-1140, 1999.

62. Wenning GK, Odin P, Morrish PK et al. Short- and long-term survival and function of unilateral intrastriatal dopaminergic grafts in Parkinson's disease. Ann Neurol 42:95-107, 1997.

63. Brundin P, Pogarell O, Hagell P et al. Bilateral caudate and putamen grafts of embryonic mesencephalic tissue treated with lazaroids in Parkinson's disease. Brain 123:1380-1390, 2000.

64. Remy P, Samson Y, Hantraye P et al. Clinical correlates of [18F]fluorodopa uptake in five grafted parkinsonian patients. Ann Neurol 38:580-588, 1995.

65. Hauser RA, Freeman TB, Snow BJ et al. Long-term evaluation of bilateral fetal nigral transplantation in Parkinson disease. Arch Neurol 56:179-187, 1999.

66. Kordower JH, Freeman TB, Chen EY et al. Fetal nigral grafts survive and mediate clinical benefit in a patient with Parkinson's disease. Mov Disord 13:383-393, 1998.

67. Freed CR, Greene PE, Breeze RE et al. Transplantation of embryonic dopamine neurons for severe Parkinson's disease. $N$ Engl $J$ Med 344:710-719, 2001.

68. Olanow CW, Goetz CG, Kordower JH et al. A double-blind controlled trial of bilateral fetal nigral transplantation in Parkinson's disease. Ann Neurol 54:403-414, 2003

69. Gill SS, Patel NK, Hotton GR et al. Direct brain infusion of glial cell line-derived neurotrophic factor in Parkinson disease. Nat Med 9:589-595, 2003.

70. De La Fuente-Fernandez R, Pal PK, Vingerhoets FJG et al. Evidence for impaired presynaptic dopamine function in parkinsonian patients with motor fluctuations. J Neural Transm 107:49-57, 2000 . 
71. Playford ED, Brooks DJ. In vivo and in vitro studies of the dopaminergic system in movement disorders. Cerebrovasc Brain Metab Rev 4:144-171, 1992.

72. Rinne UK, Laihinen A, Rinne JO et al. Positron emission tomography demonstrates dopamine D2 receptor supersensitivity in the striatum of patients with early Parkinson's disease. Mov Disord 5:55-59, 1990.

73. Antonini A, Schwarz J, Oertel WH et al. [11C]raclopride and positron emission tomography in previously untreated patients with Parkinson's disease: influence of L-dopa and lisuride therapy on striatal dopamine D2-receptors. Neurology 44:1325-1329, 1994.

74. Turjanski N, Lees AJ, Brooks DJ. PET studies on striatal dopaminergic receptor binding in drug naive and L-dopa treated Parkinson's disease patients with and without dyskinesia. Neurology 49:717-723, 1997.

75. Kishore A, De la Fuente-Fernández R, Snow BJ et al. Levodopainduced dyskinesias in idiopathic parkinsonism (IP): a simultaneous PET study of dopamine D1 and D2 receptors. Neurology 48:A327, 1997.

76. Breier A, Su TP, Saunders R et al. Schizophrenia is associated with elevated amphetamine-induced synaptic dopamine concentrations: evidence from novel positron emission tomography method. Proc Natl Acad Sci USA 94:2569-2574, 1997.

77. Torstenson R, Hartvig P, Långström B et al. Differential effects of levodopa on dopaminergic function in early and advanced Parkinson's disease. Ann Neurol 41:334-340, 1997.

78. De La Fuente-Fernandez R, Lu JQ, Sossi V et al. Biochemical variations in the synaptic level of dopamine precede motor fluctuations in Parkinson's disease: PET evidence of increased dopamine turnover. Ann Neurol 49:298-303, 2001.

79. Henry B, Brotchie JM. Potential of opioid antagonists in the treatment of levodopa-induced dyskinesias in Parkinson's disease. Drugs Aging 9:149-158, 1996.

80. Nisbet AP, Foster OJF, Kingsbury A et al. Preproenkephalin and preprotachykinin messenger-RNA expression in normal human basal ganglia and in Parkinson's disease. Neuroscience 66:361376, 1995.

81. Jolkkonen J, Jenner P, Marsden CD. L-dopa reverses altered gene expression of substance $\mathrm{P}$ but not enkephalin in the caudate-putamen of common marmosets treated with MPTP. Mol Brain Res 32:297-307, 1995.
82. Lavoie B, Parent A, Bedard PJ. Effects of dopamine denervation on striatal peptide expression in parkinsonian monkeys. Can J Neurol Sci 18:373-375, 1991.

83. Piccini P, Weeks RA, Brooks DJ. Opioid receptor binding in Parkinson's patients with and without levodopa-induced dyskinesias. Ann Neurol 42:720-726, 1997.

84. Whone AL, Rabiner EA, Arahata Y et al. Reduced substance P binding in Parkinson's disease complicated by dyskinesias: an F-18-L829165 PET study. Neurology 58(Suppl 3):A488-A489, 2002.

85. Korczyn AD. Dementia in Parkinson's disease. J Neurol 248(Suppl 3):III1-4, 2001.

86. Miletich RS, Chan T, Gillespie M et al. Contralateral basal ganglia metabolism is abnormal in hemiparkinsonian patients. An FDGPET study. Neurology 38:S260, 1988.

87. Wolfson LI, Leenders KL, Brown LL, Jones T. Alterations of regional cerebral blood flow and oxygen metabolism in Parkinson's disease. Neurology 35:1399-1405, 1985.

88. Eidelberg D, Moeller JR, Dhawan V et al. The metabolic topography of parkinsonism. J Cereb Blood Flow Metab 14:783-801, 1994.

89. Eidelberg D, Moeller JR, Ishikawa T et al. Assessment of disease severity in Parkinsonism with fluorine-18-fluorodeoxyglucose and PET. J Nucl Med 36:378-383, 1995.

90. Peppard RF, Martin WRW, Guttman M et al. The relationship of cerebral glucose metabolism to cognitive deficits in Parkinson's disease. Neurology 38(Suppl 1):364, 1988.

91. Kuhl DE, Metter EJ, Benson DF. Similarities of cerebral glucose metabolism in Alzheimer's and Parkinsonian dementia. J Cereb Blood Flow Metab 5:S169-S170, 1985.

92. Bohnen NI, Minoshima S, Giordani B et al. Motor correlates of occipital glucose hypometabolism in Parkinson's disease without dementia. Neurology 52:541-546, 1999.

93. Hu MT, Taylor-Robinson SD, Chaudhuri KR et al. Cortical dysfunction in non-demented Parkinson's disease patients: a combined (31)P-MRS and (18)FDG-PET study. Brain 123:340-352, 2000.

94. Walker Z, Costa DC, Walker RW et al. Differentiation of dementia with Lewy bodies from Alzheimer's disease using a dopaminergic presynaptic ligand. J Neurol Neurosurg Psychiatry 73:134-140, 2002. 